\title{
La dimensión mediterránea de la Conferencia sobre la Seguridad y la Cooperación en Europa (CSCE): de región limítrofe a campo de aplicación
}

\section{Nora Sainz Gsell}

Profesora de Relaciones Internacionales

Invescigadota del Centre d'Estudis sobre la Pau i el Desarmament

Universitat Autónoma de Barcelona. 08193 Bellaterra (Barcelona). Spain

\section{Resumen}

El artfoulo pretende analizar la dimensión mediterránea de la CSCE desde su creación hasta la actualidad (1972-1994). En primer lugar, se examinan las características particulares de este proceso diplomático en el cual se inserta la cuestión mediterránea, a continuación los debates que dan lugar a su creación y las políticas que genera; para concliuir con el estudio de su evolución y desarrollo en el marco de las reuniones CSCE.

Palabras clave: CSCE-OSCE, diplomacia multilateral, seguridad, cooperación, Mediterráneo.

Abstract. The Mediterranean dimension of the CSCE. From bordering region to implementation area

The purpose of this article is to analyze the mediterranean dimension of CSCE's process (1972-1994). Firstly we study the special feature of this diplomatic process. Secondly, the process of creating the mediterranean dimension and finally, we study the development of this dimension in the context of CSCE's meetings.

Key words: CSCE-OSCE, multilateral diplomacy, security, cooperation, Mediterranean region.

\section{Sumario}

Introducción $\mathrm{La} C \mathrm{CSE}$ y el Mediterráneo: el desartollo de la dimensión

La diplomacia multilateral: el peculiar proceso de la CSCE meditertánea de la CSCE (1975-1994)

La creación de la dimensión mediterránea de la CSCE

(1972-1975)

A modo de conclustón

Anexo: Estados participantes en la CSCE Bibliografía 


\section{Introducción}

El Mediterráneo ha ocupado un lugar destacado en el ámbito de la Conferencia sobre la Seguridad y la Cooperación en Europa (CSCE) ${ }^{1}$. Lo ha hecho desde una doble condición. La primera, ser la única región - mar- ${ }^{2}$ que se transforma en una dimension particular dentro de un ejercicio diplomático de ámbito paneuropeo como es la CSCE. La segunda, como manifestación tácita de la indivisibilidad de los intereses de Europa y los del Mediterráneo, vinculando la seguridad europea a la seguridad mediterránea.

La seguridad y la cooperación en el Mediterráneo aparecen como temas relacionados a la agenda de la CSCE desde los inicios de la Conferencia. Su incorporación se debe a la acción sostenida y persistente de algunos países mediterráneos miembros de la CSCE — como es el caso de Malta -, que hacen de los problemas del Mare Nostrum sus objetivos de políticas exteriores. Estas políticas, no obstante, serán motivo constante de tensión y discordia entre los participantes, llevando a la creación de verdaderos lobbies, en pro y en contra de la cuestion mediterránea, en el seno de la Conferencia.

El presente trabajo pretende analizar la dimensión mediterránẹa de la CSCE y su desarrollo posterior en el marco de la Conferencia. A tal fin, abordamos en primer lugar las características peculiares del proceso diplomático de la CSCE, contexto en el que se incribirá la dimensión mediterránea. A continuación, se analizan la creación de la mencionada dimensión y los distintos puntos de vista y agrupamientos de actores que la cuestión mediterránea generó dentro del proceso CSCE. Finalmente, se examinará el desarrollo del capínulo Mediterráneo en el seno de dos tipos de reuniones CSCE, las de Continuidad y los seminarios especializados en la cuestión.

\section{La diplomacia multilateral: el peculiar proceso de la CSCE}

La CSCE nace en 1972 como espacio de diálogo entre el Este y el Oeste. Hasta finales de 1990 se la considera una experiencia única y original en el contexto de las relaciones diplomáticas. No pudiéndola definir como una organización internacional, puesto que no tenia instituciones permanentes, ni tampoco como una conferencia propiamente dicha, ya que no poseía regularidad fija, el concepto que más se aproximaba y que mejor describía y explicaba la CSCE era el de proceso. De ahí que se la haya definido como "proceso multilateral de negociaciones continuas y conjuntas sobre cuestiones relativas a la seguridad y a la cooperación en Europan (Ghebali, 1989: 3). Estas negociaciones reunieron a los Estados pertenecientes a la Organización del Tratado del Atlántico Norte (OTAN) y a los del Tratado de Varsovia (TV),

1. A partir del primero de enero de 1995, la Conferencia sobre la Seguridad y la Cooperación en Europa (CSCE) se denomina Otganización para la Seguridad y la Cooperación en Europa (OSCE).

2. Los otros mares de ese espacio paneuropeo son: el mar del Norte, el Báltico y el Atlántico. 
como también a aquéllos fuera de las alianzas militares, es decir, los neutrales $y$ no alineados (NNA) ${ }^{3}$.

Sin embargo, su peculiaridad no derivaba solamente de su calidad de proceso, sino especialmente de su metodología de trabajo, caracterizada por:

- la agrupación temática de las cuestiones a tratar en la conferencia en tres grandes "cestos" o "canastas": a) las cuestiones relativas a la seguridad; b) la cooperación en materia de economía, ciencia y tecnología, y medio ambiente; y c) la cooperación en el ámbito de los derechos humanos;

- la participación de los Estados sobre la base de una plena igualdad (fuera de las alianzas militares y de agrupaciones políticas) y

- la utilización del consenso ${ }^{4}$ para la adopción de decisiones.

Estas dos últimas características, igualdad y consenso, colocan a los pequeńos Estados al mismo nivel que las grandes potencias en las negociaciones diplomáticas. Y en lo relacionado con la cuestión mediterránea, esto jugará un papel clave, como veremos más adelante.

Producto de este conjunto de negociaciones en el marco de la CSCE fue la firma, en agosto de 1975, del Acta Final de Helsinki. El Acta, con su Decálogo de principios que rigen las relaciones entre los Estados participantes, se convirtió en un verdadero "código de conducta" para los países signatarios.

Este proceso sin instituciones ha tenido en las Reuniones de Continuidad o de Seguimiento de la CSCE su eje vertebrador. Dichas reuniones tienen como objetivos básicos evaluar la puesta en práctica de las disposiciones del Acta Final y profundizar y fijar el programa de trabajo de la CSCE. Entre 1975 y 1994 han tenido lugar cinco Reuniones de Continuidad: Belgrado (1977-1978); Madrid (1980-1983); Viena (1986-1989); Helsinki (1992) y Budapest (1994). Mediante la celebración de reuniones anexas (conferencias, seminarios, foros especializados, etc.) la CSCE ha desarrollado su programa de actividades.

Como consecuencia de los cambios acaecidos en el escenario europeo, los jefes de Estado o de Gobierno de los países participantes en la CSCE firmaron la Carta de París para una Nueva Europa (CP) en noviembre de 1990. Este documento ponia fin a la división de Europa y señalaba la desaparición de los bloques. La CP aspiraba a la "recreación» de Europa en base a la democracia, la paz y la unidad, apareciendo la CSCE como el foro idóneo para llevar a cabo esos objetivos. Sin embargo, para ello debia desarrollar estructuras y crear

3. En este diálogo participaron todos los Estados europeos a excepción de Andorra y Albaniz (ésta úfrima se incorporaría en 1991). Ver listado anexo y actualizado de países partictpantes en la CSCE.

4. El consenso es definido como "la ausencia de cualquier objeción expresada por un representante y presentada por él como obstáculo para la adopción de la decisión de que se traten. RFCH, cap. 6, párr. 69. 
organismos, lo que implicaba su gradual transformación en organización internacionals.

Dentro de este proceso, las cuestiones mediterráneas han ocupado un lugar que podemos calificar, al mismo tiempo, de sustancial y de marginal. En el primer caso, el calificativo obedece a que la cuestión es generadora de debates. En el segundo, a que como espacio de diálogo euromediterráneo, productor de seguridad y de cooperación, se puede describir como simulacro de diálogo.

\section{La creación de la dimensión mediterránea de la CSCE (1972-1975)}

El Mediterráneo, región caracterizada por su alto grado de conflictividad ${ }^{6}$, fue un tema que generó diferentes actitudes en los Estados implicados en el proceso CSCE, despertando - según Fuentes (1989: 53) — recelos en las grandes potencias, indiferencia en los participantes no ribereños, interés en los costeros $y$ pasión en Malta.

La inserción de la "cuestión" mediterránea, de naturaleza Norte-Sur, dentro del proceso $\mathrm{CSCE}$, concebido como foro de negociaciones entre el Este y el Oeste, reveló desde el inicio de la Conferencia diferentes posiciones de partida entre los convocados a participar. A priori, podermos identificar dos, basadas en un cleavage tradicional en el escenario europeo, el Norte-Sur. Por un lado, la posición de los europeos del norte - Suecia, Noruega, Dinamarca-, mediatizados por la «lejanía de la cuestión, que pretendían que la conferencia se centrase exclusivamente en los problemas de las relaciones Este-Oeste ${ }^{7}$. Posición a la que se sumaban Estados Unidos y la antigua Unión Soviética.

5. El marco institucional conformado por la Carta de Parfs comprende un Secreariado, un Centro de Prevención de Conflictos, una Asamblea Parlamentaria y una Offcina de Elecciones Libres (rransformada, más tarde, en Oficina de las Instituciones Democtáticas y de los Derechos Humanos). Se establecen también una serie de mecanismos de consulta política: un Consejo de Ministros, un Comité de Altos Funcionarios, la reunión periódica de los Jefes de Estado o de Gobierno y se mantienen las teuniones de Continuidad de la conferencia, ahora con carácter regular. Este marco institucional se ve incrementado con la creación de las figuras del Alto Comisionado de las Minorias Nacionales (DRH, cap. I, párr. 23) y la del Secretario General (Decistones del Consejo de Estocolmo, 1992, cap.I, párr. 7).

6. Como ha quedado de manifiesto a lo largo de los distintos artífulos de este monográfico, la problemática del Mediterráneo riene múltiples dimensiones de análisis (histórico-culturales, políricas, económicas, religiosas, medioambientales, erc.). En la época que se convoca y se desarrolla la CSCE, af́os setenta, la región mediterránea, definida en su sentido más amplio, exteriorizaba un alto grado de conflictividad (el conflicto árabe-israelf en su extremo oriental, la cuestión de Chipre en su zona central y el problema del Sahara en su región occidental).

7. La CSCE se convocó a fin de alcanzar algún tipo de acuerdo sobre los problemas europeos heredados del fin de la segunda guerra mundial. A los páses del bloque del Este les inceresaba legitimar las fronteras surgidas, statu quo territorial. Los del Oeste, sin objetivos claros en un principio, se centrarian paulatinamente sobre la cuestión de los derechos humanos. 
Por otro lado, la de los europeos del sur, próximos a la cuestión, principalmente España, Yugoslavia y Malta, que consideraban que los temas de cooperación y seguridad en Europa debían complementarse con su vertiente mediterránea. De esta forma quedaban plasmadas percepciones distintas de los problemas europeos, que se traducirían en el debate sobre la oportunidad de crear una dimensión mediterránea en el contexto CSCE. Antes de introducirnos en el debate concreto es necesario abordar la constitución de agrupaciones de Estados, que presionarán en uno u otro sentido.

\section{Los grupos}

En los inicios de las negociaciones que darían lugar a la Conferencia, hay que destacar las "presiones exógenas" a la misma, es decir, las ejercidas por Estados mediterráneos no europeos. Dos países en particular - Argelia y Túnez- se manifestarían los más activos en este sentido ${ }^{8}$, solicitando su participación en la futura CSCE. Ambos fundamentarían su posición en la existencia vínculos históricos, económicos y políticos entre las dos riberas de la cuenca mediterránea. Los argumentos del "lobby norteafricano" no caerán en saco roto, como afirma, Ghebalí (1989: 364), puesto que reforzarán la postura del primer conjunto de Estados: los que conforman el ala pro-mediterránea de la Conferencia. Sector que se caracterizará por el juego de tres grupos de actores.

En primer término, lo que se ha denominado el "frente avanzado" de la dimensión mediterránea, constituido por Chipre, Malta y la antigua Yugoslavia (mediterráneos no alineados). Los objetivos de este grupo, amén de los matices individuales, iban encaminados a obtener que los beneficios de la distensión se extendiesen al Mediterráneo. Invocando la inseparabilidad de los intereses económicos y de seguridad de la cuenca mediterránea de los principios de seguridad y cooperación en Europa. A la acción de estos paises hay que sumar la de España, que en los primeros tiempos de la CSCE llevaría a cabo una activa política de apoyo a la participación de los mediterráneos no euro$\operatorname{peos}^{2}$.

8. Con motivo de las conversaciones prepararorias de la Conferencia, en Dípoli (1972), Argelia y Túnez hicieron circular documentos, manifestando su inequivoca "vocación europea", vinculando su futuro más con Europa que con el mundo árabe (Maresca, 1987:189). Argelia fue el pais que más activo se mostró en este sentido. Aprovechó cualquier foro diplomático (Naciones Unidas, No Alineados, etc.) para hacer saber que se sentía directamente concernida en una negociación paneturopea, que tratase de la seguridad y la cooperación en Europa.

9. Hasta su adhesión a la OTAN en 1982, España formaba parte del grupo de actores más hererogéneo de la CSCE, el denominado genéricamente NNA. Este bloque agrupaba a páses neutrales (Austria, Finfandia, Suecia y Suiza); a Estados pertenecientes al Movimiento de No Alineados (Chipre, Maita y la antigua Yugoslavia); a micro estados, que no eran ni neutrales ni no alineados (Liechtenstein, Mónaco y San Marino) y los independientes (España y la Santa Sede). Los temas que la dipłomacia española presentó a la Conferencia hacian referencia a las siguientes cuestiones: Gibraltar, el Mediterráneo, el turismo y la emigración. 
A continuación, el conjunto formado por los Estados mediterráneos pertenecientes a la Comunidad Europea y/o a la OTAN, Grecia, Francia, Italia y Turquía. El interés principal de este grupo era aprovechar la buena disposición de los mediterráneos de la ribera norte para dialogar sobre problemas comunes, en particulat económicos ${ }^{10}$. En especial franceses e italianos utilizarán esta circunstancia como oportunidad política para afirmar y dar credibilidad a la política mediterránea de la Comunidad hacia este marco (véase artículo de Barbé).

Finalmente, constituyendo la retaguardia del ala pro-mediterránea, Austria, Rumanía y Suiza, europeos no mediterráneos; que se muestran favorables a incorporar la cuestión mediterránea a la CSCE. Esta postura, sostenida en especial por austríacos, pretende que Europa desempeñe algún papel constructivo (mediador, buenos oficios) en un problema que está presente y condicionando las conversaciones preparatorias de la CSCE: el conflicto árabe-israelí.

La guerra que enfrenta al mundo árabe con Israel e intereses nacionales de política exterior, aparecen como los elementos aglutinantes de la segunda agrupación de Estados: los que se opondrán a la existencia de la dimensión mediterránea en el marco CSCE. De manera general, todos los países contrarios a la cuestión mediterránea, temían que la introducción del conflicto árabe-israelí (considerado como periférico) comprometiese el diálogo entre el Este y el Oeste, que tanto había costado convocar. A nivel específico de los Estados, todos tenían objetivos concretos. Para EEUU y Gran Bretaña la creación de una vertiente mediterránea en la CSCE implicaba legitimar la presencia soviética en el Mediterréaneo. Para la URSS, era un problema Norte-Sur y debía permanecer dentro del ámbito de Naciones Unidas. Para los paises del Este, sólo se convertiría en un problema que ralentizaría las discusiones, y para los escandinavos (los más hostiles a la cuestión) no era ni siquiera susceptible de ser sugerida. Para todos ellos sólo una cuestión estaba clara: la CSCE debía desarrollarse en un estricto marco Este-Oeste.

Los distintos posicionamientos sobre el tema Mediterráneo evidenciaban que éste se había convertido en fuente de tensión entre los participantes. De allí que se decidiera incluirlo --aunque de manera difusa - en el orden del día de la agenda de la Conferencia ${ }^{11}$. De esta forma comienza a cobrar cuerpo una incipiente dimensión mediterránea en la CSCE, que generará un delicado debate en torno a ella.

10. En el memorándum argetino de 1972 aparecen los términos de complementariedad y solidaridad en materia de flujos económicos, cometciales y de politica energética, entre el Magreb y la entonces Comunidad Económica Europea (CEE) (para más información sobre Magrebeconomia, véase el artículo de Mir).

11. El tema es incluido de la siguiente manera: wal considerar las cuestiones relacionadas con la seguridad en Europa, se tendrá en cuenta el contexto más amplio de la seguridad mundial y, en particular, la relación que existe entre la seguridad en Europa y en la región del Mediterráneo", RFCH, cap. 2, párr. 15. 


\section{El debate}

La discusión sobre la cuestión mediterránea versó sobre dos temas esenciales. Por un lado, la calidad de participación de los Estados mediterráneos no europeos y, por otro, la aplicación de las disposiciones surgidas en el seno de la Conferencia a la región mediterránea.

El primer tema, la definición del estatuto de los no europeos de la región mediterránea, generó propuestas muy dispares. Entre los «mediterranistas» el espectro va desde opciones maximalistas como la maltesa, que propone la participación de estos Estados en pie de igualdad con el resto de los convocados; a posiciones minimalistas como la de Rumanía, que sugiere se tome nota de las opiniones de los Estados no participantes sobre los diferentes temas del orden del día. En el lado de los contrarios a la cuestión, el tema se limitaba a una única posibilidad: la exclusión de los mediterráneos no europeos (Maresca, 1987: 190). Como arreglo que satisfaciese ambas posiciones, se decidió otorgarles un estatuto formai de estados mediterráneos no participantes (EMNP). Denominación ambigua puesto que no les autoriza a participar plenamente en los trabajos de la CSCE, y los sitúa en una categoría inferior a la de observadores. A escos EMNP sólo se les permitiría asistir a ciertas reuniones de la CSCE, las de Continuidad y las relativas a La cooperación en el Mediterráneo. En ellas presentan sus puntos de vista respectivos por medio de exposiciones orales y escritas denominadas contribuciones (los participantes de pleno derecho formulan proposiciones).

Una vez establecida la categoría de EMNR, surgirían los interrogantes: ¿qué países podían ser incluidos en ella?, ¿qué Estados tendrían derecho a ser oídos en un foro que reúne a la mayor parte de los Estados desde Vancouver a Vladisvostok? Se consideraba Estados no participantes a los «situados en las regiones adyacentes a Europa, en particular a aquellos Estados mediterráneos que han expresado ya su interés en manifestar sus puntos de vista a la Conferencia» (RFCH, cap. 3, párr. 57). En esta definición se hacía referencia a argelinos y tunecinos, no obstante otros mediterráneos manifestarán sus deseos de participar en la CSCE. Así, Egipto, Marruecos, Siria e Israel ${ }^{12}$ enviarán representantes. La participación de este último país abriría una nueva vía en el debate en torno a los EMNP, no sólo por la participación de los israelíes, sino también por la actitud maltesa de negarles la palabra, haciendo uso de un instrumento de la propia Conferencia: el consenso. Malta instrumentalizaría la regla del consenso bloqueando sistemáticamente todo aquello que consideraba iba en contra de sus intereses, llegando a hacer del chantaje del consenso una constante de su actuación en la CSCE, que puso en peligro la continuación

12. Libia y Líbano no enviaron representantes a esta primeras fases de la Conferencia. El primero porque consideraba al proceso CSCE como lejano a sus intereses. El segundo porque ya vivja inmerso en un clima de pre-guerra. Ambos países se incorporarían con posterioridad a la CSCE. Lfbano con motivo de la Reunión de Belgrado (1977) y Libia en la Conferencia de Estocolono (1984). 
de la misma ${ }^{13}$. La presencia israelí era para los malteses - que han llevado a cabo una clara política pro-árabe en el seno de la CSCE- un elemento pertubador en sus objetivos de actuación exterior. La situación logra salvarse gracias a la acción moderadora de otros países, tanto simpatizantes de la causa árabe (España), como con claras simpatías hacia Israel (Canadá, Holanda y Dinamarca) ${ }^{14}$.

El segundo tema, la aplicación de las disposiciones surgidas en la CSCE a la región mediterránea, está en estrecha relación con la creación formal de la dimensión mediterránea de la CSCE. Solucionado el problema de la forma de participación de los EMNP, surgía el dilema de incluir en el documento de conclusión de las negociaciones -Acta Final de Helsinki (AF) - un apartado que hiciese mención al Mediterráneo, lo que implicaría la única regionalización de la Conferencia. En este terreno surgirán, grosso modo, tres grandes opciones. El ala mediterránea más radical - Malta, Chipre, Yugoslavia- postula la necesidad de crear un capítulo autónomo dentro del $\mathrm{AF}$, que trascendiese las apariencias y vinculase la seguridad y la cooperación en Europa con la de la región mediterránea (Maresca, 1987: 190). Desde las filas del atlantismo -EEUU, Gran Bretaña- se contempla la idea con desconfianza, puesto que implica distorsionar el carácter Este-Oeste de la Conferencia e inscribir de forma permanente la cuestión mediterránea en la agenda de la CSCE. EI tercer grupo de palses - España y particularmente los mediterráneos comunitarios- se muestran partidarios de una solución a medio término entre una y otra posición. La CSCE debe producir una declaración sobre el Mediterráneo, inserta en el AF, siendo su contenido aceptable pero no vinculante en los temas de fondo (Maresca, 1987: 191). Esta iniciativa de origen italiano, y adoptada posteriormente por los nueve miembros de la Comunidad Europea, es la que al final se impondrá, siendo fundamental la gestion llevada a cabo por Alemania, encargada de la presidencia comunitaria 15 .

De esta forma nacia formalmente la dimensión mediterránea de la CSCE. Todos los temas relacionados a ella se recogían en el capítulo del AF denominado Cuestiones relativas a la seguridad y a la cooperación en la región del Mediterráneo, de mero carácter declatatorio. El contenido del capítulo "Meditertáneo" ya nos da indicio del posible alcance de las disposiciones surgidas en el seno de la CSCE al ámbito meditertáneo.

13. La intransigencia de Malta en la utilización del consenso fue tal, que la hoy desaparecida URSS llegó a sugetir durante las negociaciones que el proceso CSCE se realizase sin la participación maltesa (Fuentes, 1989: 53).

14. Los EMNP fueron "apadrinados" por otros Estados participantes en la CSCE. Así, Marruecos lo fue por España y Francia; Egipro por Yugosiavia; Siria por Malra, e Israel por Dinamarca y Holanda. La exclusión de Israel de los EMNP había sido sostenida por Yugoslavia y por la URSS, aparte de Malta, pero no con la virulencia de esta última.

15. Hans D. Genscher, ministro alemán de AA.EE., le solicita personalmente a Henry Kissinger, secretario de Estado, que EEUU no bloquee la iniciativa de los Nueve (Maresca, 1987: 191). Esta entrevista es una de las pocas acciones alemanas vinculadas al Mediterránto en la CSCE. 
Si consideramos la cuestión seguridad (aspectos político-militares), aparecida en el AF, y en particular el Documento sobre las Medidas destinadas a fomentar la confianza y ciertos aspectos de la seguridad (MDC), no se encuentra más que una referencia vaga a la región, limitando las relaciones de los ribereños a las de "buena vecindad", quedando este apartado reducido al marco Este-Oeste. Si observamos la cuestión cooperación, ésta se reduce a confirmar la existencia de intereses comunes en el campo de la economía, de la industria y de la ciencia, pero no admite la interdependencia de ningún tipo entre Europa y el Mediterráneo, según Ghebali (1989: 371).

El contenido del AF, a nivel global, y de la Declaración sobre el Mediterráneo, de manera específica, muestran una dimensión vacía de seguridad y pobre de cooperación.

Si bien la creación de la dimensión mediterránea de la CSCE constituía el primer ensayo de diálogo euro-árabe sobre los problemas del Mediterráneo ${ }^{16}$, nacía, de partida, con elementos que condicionarían su desarrollo y funcionamiento. Por un lado, la marginalización de los EMNP en el seno de la Conferencia y, por otro, la no aplicación a la región mediterránea de las disposiciones surgidas en la CSCE.

En el primer caso, se les habia ortogado un estatuto incierto, EMNP, cuyo fin último era evitar que los actores del conflicto árabe-israelí (de naturaleza Norte-Sur) pudieran ejercer un derecho de veto (a través de la regla del consenso, por ejemplo) sobre los problemas Este-Oeste. En el segundo, no se admitía que regímenes como los de las MDC de Helsinki y más tarde el de MDCS de Estocolmo se extendieran a la cuenca mediterránea, puesto que implicaba constreñimientos a la política de las grandes potencias ${ }^{17}$. No obstante estas limitaciones, la dimensión mediterránea de la CSCE cobraba vida.

\section{La CSCE y el Mediterráneo:}

\section{el desarrollo de la dimensión mediterránea de la CSCE (1975-1994)}

Ei desarrollo de la dimensión mediterránea de la CSCE será analizado en el marco de las Reuniones de Continuidad de la Conferencia y en el de los seminarios especializados en materia de cooperación, únicos espacios de encuentros entre los Estados participantes y los EMNP de la CSCE.

16. La dimensión mediterránea de la CSCE es el primer ejercicio diplomácico multilateral en tratar los problemas concernientes al Mediterráneo. Luego vendrían otros como el Diálogo Euro-árabe (1974) entre los paises de la CE y los de la Liga Árabe y las Conferencias Ministeriales sobre el Mediterráneo de los países No Alineados (1984). Con la desaparición de los bloques, ha habido varios intentos de diálogo como la fallida CSCM o el Diálogo Cinco más Cinco (véase el artículo de Barbé).

17. Los regímenes de MDC y de MDFS hacian referencia sólo a medidas político-militares de carácter terrestre y no marítimas. Esta posición fue sostenida por los miembros de la OTAN, para evirar cualquier tipo de limitación a sus fuerzas navales en el Mediterráneo. 


\section{Reuniones de Continuidad de la CSCE}

Las Reuniones de Continuidad de la CSCE (también llamadas de seguimiento) incluidas como capítulo dentro del AF verán marcado su desarrollo por los acontecimientos acaecidos en el sistema internacional.

La primera de ellas tendrá lugar en Belgrado (1977-1978) y los trabajos se desarrollarán bajo los efectos del fin de la distensión. En la capital de la antigua Yugoslavia se evaluará por vez primera la aplicación de las disposiciones del AF. La cuestión mediterránea se caracterizará, por un lado, por la actuación de los EMNP y, por otro, como en las negociaciones anteriores, por el proceder de la diplomacia maltesa ${ }^{18}$ (el factor maltés), que se convertirá en uno de los elementos claves en el desarrollo de la cuestión. Los EMNP solicitarán una participación más activa, menos episódica y más efectiva (Ghebalf́, 1989: 374). Sin embargo, el conflicto de Oriente Medio impregna el desarrollo del encuentro y se opta por opciones continuistas: mantener la política de marginalización. Por su parte, Malta, siempre desde posturas maximalistas, pretende la institucionalización de la dimensión mediterránea a través de la creación de un comité permanente sobre la seguridad y la cooperación en el Mediterráneo. La intransigencia maltesa vuelve a colocar a la Conferencia al borde de sus posibilidades. In extremis, los europeos mediterráneos - Francia, Italia (al margera de las posturas comunitarias) y España - encuentran una fórmula que evita el fracaso: la convocatoria de una reunión en La Valetta, capital maltesa, con el fin de uexaminar las posibilidades y medios de fomentar iniciativas concretas para una cooperación mutuamente ventajosa en las diversas esferas de la economía, la ciencia y la cultura" (CSCE/DRBL).

La Reunión de Madrid (1980-1983) se desarrollará en una situación internacional marcada por el triángulo de acontecimientos Kabul-Varsovia-Seúl ${ }^{19}$. El capítulo Mediterráneo vivirá por enésima vez el psicodrama maltés, que en esta ocasión alcanzará su punto culminante. Malta intenta conseguir una conferencia mediterxánea de seguridad y la extensión de las MDC al ámbito mediterráneo. Sus exigencias extremas, respaldadas por el chantaje del consenso, ponen en actividad al ala moderada de los mediterráneos. Esta, encabezada por la diplomacia espaniola, logra un documento final en el cual los Estados participantes manifiestan a fortiori su vóluntad de "adoptar medidas positivas para la disminución de las tensiones y el fortalecimiento de la estabilidad, seguridad y paz en el Mediterráneo[...]" (CSCE/DRM, Cuestiones relativas a la

18. Malta, antigua colonia británica, pás históricamente, étnicamente y económicamente vinculado al norte de África, se siente mediterzánea antes que europea. El entonces primer ministro maltés, Dom Mintoff, afirmó en el seno de la Reunión de Belgrado «el Mediterráneo es el único objetivo de nuestra política exterior y Malta está dispuesta a enfrentarse con Europa a causa del mar tantas veces como sea necesarion (Fuentes, 1989: 106).

19. Los hechos más significarivos ocurridos en paralelo a la reunion de Continuidad de Madrid fueron: la cuestión de la invasión soviética a Afganistán, la situación polaca que daría lugar al golpe militar de 1981 y el derribo de un avión surcoreano por efectivos soviéricos. A esto hay que agregar la crisis de los euromisiles que agudizaba las tensas relaciones Este-Oeste. 
seguridad y la cooperación en el Mediterráneo), acordándose la reunión de un encuentro sobre la región en Venecia.

La Reunión de Continuidad de Viena (1986-1989) se verá totalmente influida por la política de la perestroika. Así pues, el efecto Gorbachov y la ausencia del factor maltés ${ }^{20}$ acentuarán aún más las carácterísticas Este-Oeste del proceso CSCE. No obstante, el documento de clausura recogerá el deseo de los treinta y cinco Estados de convocar una reunión sobre la región del Mediterráneo a fin de "considerar vías y medios de llevar más adelante los diferentes aspectos de la cooperación, incluida la protección y el mejoramiento de los ecosistemas mediterráneos a fin de [...]contribuir al fortalecimiento de la confranza y de la seguridad en la región» (CSCE/DRV). Se estipula que el encuentro se celebre en Palma de Mallorca.

En Helsinki (1992), tendrá lugar la primera reunión de Continuidad de la CSCE en un nuevo contexto internacional, el del fin del bipolarismo, y con una CSCE institucionalizada. Los temas mediterráneos, discutidos casi marginalmente en las sesiones oficiales, son el centro de las conversaciones informales, tanto de los EMNP como de los europeos mediterráneos. El frente mediterráneo -encabezado por España y Malta y secundado por Francia, Italia, Grecia, Turquía y Chipre ${ }^{21}$ - transformado en el grupo «amigos del Mediterráneo" (Heraclides, 1992: 162), será el generador de una serie de propuestas encaminadas a la intensificación de la cooperación con los EMNP22. El grupo llega a sugerir la convocatoria de un foro mediterráneo (a imitación del Foro Económico $\mathrm{CSCE}^{23}$ ), cuya agenda incluiría cuestiones tales como: inmigración, medio ambiente, tendencias demográficas, desarrollo económico, etc.). Algunas de estas proposiciones son adoptadas por los Doce, quienes presentarán una propuesta común, la que con algunas variaciones aparecería reflejada en el apartado correspondiente al Mediterráneo en el documento firal de la reunión (CSCE/DRH, cap. VI, párr. 6).

Así, como incipiente producto de la desaparición de la lógica Este-Oeste, las cuestiones relacionadas con el Mediterráneo parecen querer tener cierta

20. Los cambios en la política interna maltesa, Don Mintoff deja de ser primer ministro en 1987, repercuten sobre la cuestión mediterránea en el ámbito de la CSCE.

21. El lobby meditertáneo sufre el impacto de la crisis del grupo de los NNA. La antigua Yugoslavia, uno de los pilares de la cuestión mediterránea dentro de la CSCE, vive la guerra en sus territorios y Malta y su política de no alineamiento pierde fuerza con la desaparición del eje Este-Oeste.

22. Entre 1990-1992, Francia es uno de los países más activo en la cuestión mediterránea en la CSCE institucionalizada. Por ejemplo, es el único Estado que propone algún tipo de estatuto especial para la participación de los EMNJ' en la Asamblea Parlamentaria de la CSCE, creada en abril de I991 (Propuestas para el encuentro de las delegaciones parlamentarias de los Estados participantes en la CSCE (Madrid, 2 y 3 de abril 1991), Madrid, 27 de marzo 1991.

23. El Foro Económico de la CSCE, creado en enero de 1992 (Reunión de Praga del Consejo de la CSCE, Documento sobre el ulterior desarrollo de las instituciones y estructuras de la CSCE, cap. V, párr. 19, 30-31 de enero de 1992), tiene como una de sus funciones examinar el cumplimiento de los compromisos de la CSCE en las esferas de la economía, medio ambiente y ciencia y tecnología (CSCE-DRH, cap. 7, párr. 31). 
relevancia en el ámbito de la nueva CSCE. Se reconoce al Comiré de Altos Funcionarios (CAF) - organo creado por la $\mathrm{CP}$ - como el mecanismo apropiado para tratar las cuestiones relativas a la región, otorgándole la capacidad de examinar las modalidades de contribución de los EMNP (DRH, cap. X, párr. 1, 2 y 3). Se decide convocar un seminario bajo los auspicios del CAF, cuyos temas son, casi en su totalidad, los propuestos por los "amigos del Mediterráneo" y adoptados por la CE.

La Reunión de Continuidad de Budapest (1994) producirá un documento final, innovador en lo relativo al Mediterráneo, que incluye, por primera vez en la historia de la CSCE, una referencia al tema tabú de la región: el conflicto de Oriente Medio. Aunque meramente declaratorio, se vincula la seguridad europea con la paz en esa zona (CSCE/DRBD, párr. 17). Se menciona la cooperación con algunos EMNP (no se alude a Libia ni al Líbano) como el medio de reforzar y profundizar la estabilidad en la zona de la CSCE. Otra novedad que se incluye en el documento es la celebración de un seminario sobre el Mediterráneo que tendría lugar en Egipto (Ibid., cap. X, párr. 2 (b)).

\section{Seminarios}

Las reuniones o seminarios de cooperación en cuestiones mediterráneas que han tenido lugar en el contexto de la CSCE nos permiten considerar un aspecto no tratado aún, los temas de diálogo entre los Estados del proceso CSCE.

El primer seminario tiene lugar en La Valetta (febrero-marzo 1979), como retribución a la constante acción de la diplomacia maltesa. Por presiones de la mayoría de los páses miembros de la OTAN como los del TV, el DRBL había excluido expresamente el tema de la seguridad, limitando la cooperación a campos que no generarían conflicto: la economía, la ciencia y la cultura ${ }^{24}$.

En el encuentro de Venecia (octubre 1984), la cuestión mediterránea iba a salir tal como entró, reducida a los temas de cooperación tratados con anterioridad.

La reunión llevada a cabo en Palma de Mallorca (octubre 1990) significa cierto punto de inflexión en el contenido ternático de los seminarios en materia de cooperación en el Mediterráneo. A los temas tratados con anterioridad, se suman los de carácter medioambiental, destacándose el de "la protección de los ecosistemas mediterráneos" (apareciendo por primera vez el agua como factor de cooperación entre los participantes ${ }^{25}$ ).

Este encuentro es el primero de la CSCE que se lleva a cabo dentro de un contexto internacional en plena transformación, con los cambios en la Europa

24. En el apartado economfa, se propone intensificar los intercambios tanto de carácter bilateral, como en el marco de las organizaciones internacionales competentes. En el de ciencia, la celebración de reuniones científicas sobre los problemas más apremiantes de la región. En el de la cultura, desarroliar la cooperación en ja investigación y conservación del patrimonio cultural e histórico del Mediterráneo (Documento de la Valetta, 1979).

25. Para más detalles sobre el agua como factor de conflicto y de cooperación en las relaciones internacionales véase el artículo de Izquierdo). 
Oriental y la guerta del Golfo Pérsico como hechos destacables. A pesar del conflicto que enfrenta a algunos países árabes con Israel, el seminario logra reunir a todos los EMNP. Es en el marco de este seminario cuando se hace pública una propuesta conjunta hispano-italiana sobre la convocatoria de una Conferencia sobre la Seguridad y la Cooperación en el Mediterráneo (CSCM). Dicha CSCM, ajena a la CSCE pero utilizando sus métodos de trabajo (división temática, creación de un Acta -del Mediterráneo-, codificación de valores comunes) y sus productos (creación de un régimen de MDCS mediterráneo como instrumento de prevención de crisis) pretende crear elementos de seguridad y de cooperación en el marco del Mediterráneo. Así pues, la región mediterránea aparece como zona cuyos problemas - locales y regionalesnecesitan ser tratados desde una aproximación global.

En el último encuentro sobre el Mediterráneo, realizado en La Valetta, mayo (1993), se abordarán los temas recogidos en el DRH (sugeridos por el lobby mediterráneo) con el fin de crear y fomentar medidas prácticas de cooperación con lo EMNP. Lamentablemente, los resultados no son todo lo alentadores que se desea.

La cuestión mediterránea sigue sin ser relevante. En los encuentros generales como en los de los seminarios especializados, las cuestiones sobre las que dialogar --temas-continuan provocando frustraciones, sobre todo en los países mediterráneos de la ribera sur.

\section{A modo de conclusión}

Este recorrido por los distintos aspectos del capítulo Mediterráneo de la CSCE nos permite formular una serie de apreciaciones de la dimensión mediterránea en tanto que espacio de diálogo y región generadora de políticas.

Como espacio de diálogo, los escasos resultados obtenidos a lo largo de estos años hacen de este encuentro multilateral euromediterráneo un lugar de reflexión poco sólido. Esta escasa solidez o inconsistencia de la cuestión mediterránea en el ámbito de la CSCE está motivada por distintas razones.

- En primer lugar, la voluntad manifiesta de la mayor parte de los Estados participantes en el proceso CSCE de mantener este diálogo dentro del estricto marco de las relaciones Este-Oeste.

- En segundo término, y como consecuencia de lo anterior, la aplicación de políticas de marginalización a los mediterráneos no europeos, los EMNP. Dicho de otro modo, a estos países se les ha impedido una participación efectiva, se les ha "escuchado" pero no "oído".

- Por último, la escasa relevancia de las cuestiones de agenda - los temasque han hecho que las cestas de la seguridad y de la cooperación sigan estando aún vacías.

Como región generadora de políticas, la región aunque limítrofe dentro de la CSCE, ha sido una continua creadora de "conductas" (extremas unas, moderadas otras) tal como lo hemos puesto de manifiesto. Sin embargo, desde 
el fin del bipolarismo y en el marco de una CSCE institucionalizada, el Mediterráneo comienza a ser visto ya como campo de aplicación de seguridad y de cooperación, pero no desde la CSCE, sino, quizás, por medio de la CSCE. Ello se traduce en políticas de continuidad y políticas de cambio hacia la zona.

La continuidad la observamos en el mantenimiento de constante preocupación hacia la zona por los Estados del bloque mediterráneo - englobados en su totalidad en la Unión Europea (a excepción de Turquía, Chipre y Malta)El cambio, el elemento innovador, viene del lado atlantista - principalmente de EEUU - con el "redescubrimiento" del Mediterráneo y la oportunidad que este mar ofrece para plantear "temas" dentro de la Nueva Europa. La zona comienza, también, a ser considerada como escenario de actuación futura de otras organizaciones -en especial la OTAN - y en este aspecto la CSCE podría convertirse en la macroorganización de cobertura. Paulatinamente el Mediterráneo deja de ser una región limítrofe para convertirse en una zona de aplicación de políticas ¿de la CSCE?

\section{Anexo: Estados participantes en Ia CSCE}
1) Albania
2) Alemania
3) Armenia
4) Austria
5) Azerbaidzhán
6) Belarús
7) Bélgica
8) Bosnia-Herzegovina
9) Bulgaria
10) Canadá
11) Croacia
12) Chipre
13) Dinamarca
14) Eslovaquia
15) Esiovenia
16) España
17) Estados Unidos
18) Estonia
19) Finlandia
20) Francia
21) Georgia
22) Grecia
23) Hungria
24) Irlanda
25) Islandia
26) Italia
27) Kazajstấn
28) Kirguizistán
29) Letonia
30) Liechtenstein

31) Lituania

32) Luxemburgo

33) Malta

34) Moldova

35) Mbnaco

36) Noruega

37) Países Bajos

38) Polonia

39) Portugal

40) Reino Unido

41) Rumanía

42) Federación Rusa

43) San Marino

44) Santa Sede

45) República Checa

46) Suecia

47) Suiza

48) Tadzjiskistán

49) Turkmenistán

50) Turquía

51) Ucrania

52) Uzbekistán

53) Yugoslavia (Serbia y Montenegro)*

Observaciones:

*Tiene suspendida su participación.

La antigua república yugoslava de Macedonia, parcicipa en la CSCE en calidad de observadot. Andorra no ha solicitado su incorporación. 


\section{Bibliografía}

Conferencia sobre la Seguridad y la Cooperación en Europa (CSCE).

- (1973). Recomendaciones Finales de las Consultas de Helsinki (RFCH).

- (1975). Acta Final (AF).

- (1978). Documento Final de la Reunion de Continuidad de Belgrado (CSCE/DRBL).

- (1983). Documento Final de la Reunión de Continuidad de Madrid (CSCE/DRM).

- (1989). Documento Final de la Reunión de Continuidad de Viena (CSCE/DRV).

- (1992). Documento Final de la Reunión de Continuidad de Helsinki. El Desafio del Cambio (CSCE/DRH).

- (1994). Documento Final de la Reunión de Continuidad de Budapest (CSCE/DRBD). Fuentres, Jorge (1989). El Circulo de Helsinki. Madrid: Ministerio de Asuntos Exceriores. GHEBAlI, Victor-Yves (1989). La Diplomatie de la detente. La CSCE, d'Helsinki à Vienne (1973-1989). Bruselas: Bruylant.

HERACLIDES, Alexis (1993). Helsinki-II and its Aftermath. The Making of the CSCE into an International Organisation. Londres y Nueva York: Pinter Publishers.

MARESCA, John (1987). To Helinki. The Conference on Security and Cooperation in Europe, 1973-1975. Durham y Londres: Duke University Press. 